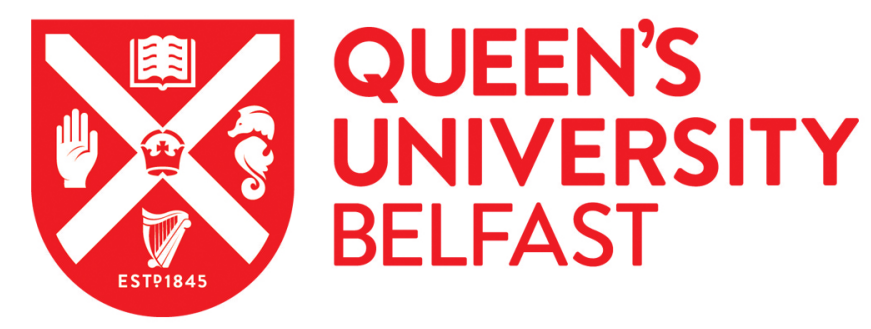

\title{
Design Guidelines on Beam Index Modulation Enabled Wireless Communications
}

Ding, Y., \& Fusco, V. (2018). Design Guidelines on Beam Index Modulation Enabled Wireless Communications. IET Microwaves, Antennas and Propagation Journal, 12(6), 993 - 998. https://doi.org/10.1049/ietmap.2017.0829

\section{Published in:}

IET Microwaves, Antennas and Propagation Journal

\section{Document Version:}

Peer reviewed version

\section{Queen's University Belfast - Research Portal:}

Link to publication record in Queen's University Belfast Research Portal

\section{Publisher rights}

(c) 2018 The Institution of Engineering and Technology. This work is made available online in accordance with the publisher's policies. Please refer to any applicable terms of use of the publisher.

\section{General rights}

Copyright for the publications made accessible via the Queen's University Belfast Research Portal is retained by the author(s) and / or other copyright owners and it is a condition of accessing these publications that users recognise and abide by the legal requirements associated with these rights.

Take down policy

The Research Portal is Queen's institutional repository that provides access to Queen's research output. Every effort has been made to ensure that content in the Research Portal does not infringe any person's rights, or applicable UK laws. If you discover content in the Research Portal that you believe breaches copyright or violates any law, please contact openaccess@qub.ac.uk. 


\title{
Design Guidelines on Beam Index Modulation Enabled Wireless Communications
}

\author{
Yuan Ding*, Vincent Fusco
}

The Institute of Electronics, Communications and Information Technology (ECIT), Queen's University of Belfast, Belfast, UK, BT3 9DT

'yding03@qub.ac.uk

\begin{abstract}
A new wireless transmission scheme, named by authors as beam index modulation (BIM), is proposed in this paper. It exploits radiation beam indices at the transmitter sides to convey information bits, in addition to the information transmitted by modulating radio frequency (RF) carriers, i.e., the classical means of wireless transmission. Favourably, only a single RF chain is required at both transmitter and receiver sides. When compared with traditional beamforming, the proposed BIM system exhibits improved bit error rate (BER) performance in sparse non-Line-of-Sight (nLoS) wireless propagation channels. A parametric study is conducted, from which the design guidelines are provided and experimentally validated.
\end{abstract}

\section{Introduction}

Multiple-input multiple-output (MIMO) techniques which exploit spatial degrees of freedom are capable of providing high transmission data rates without recourse to increase of frequency utilization and transmit power [1]. However, in practice, to construct a MIMO system, a multiplicity of associated radio frequency (RF) circuits, including RF filters, frequency mixers, and power amplifiers, are required at both transmitter and receiver sides. These components not only increase system complexity and cost substantially, but also dissipate a great amount of power, which hinders the in-field use of high-order MIMO systems in battery powered mobile applications. One possible solution to this problem uses the concept of spatial modulation (SM) [2], [3]. In SM systems, transmitters equipped with only a single RF chain can convey information bits partly through radiated RF carriers, i.e., the classical means of wireless transmission, and partly by determining the index of activated transmit antenna, which is dynamically updated based on the baseband data, at receiver side in each channel use. SM systems perform well in multipath rich environments where the channel impulse responses associated with each transmit antenna differ a lot. In these situations, SM can approach the performance achieved by full-MIMO systems. However, in sparse wireless channels that are common propagation scenarios in higher frequency bands such as millimetre wave [4], the reduced spatial degrees of freedom cannot be exploited effectively by activating only a single transmit antenna [5]. Furthermore, since with SM no beamforming gain is available, the communication link cannot be reliably maintained, especially in higher frequency bands where path loss can be huge.

In order to enable beamforming gains, at both transmitter and receiver sides, and effectively exploit spatial degrees of freedom in sparse wireless channels, while retaining the simplicity of the single RF chain architecture, the concept of beam index modulation (BIM) is described in this paper. BIM is constructed by inserting an analogue beamforming network between the antenna array and the single RF chain, at both transmitter and receiver sides. As a result of this the index of the activated antenna in the SM scheme is transformed into the index of the activated radiation beam in the BIM scheme, see Fig. 1. A related concept was recently proposed in [6], termed by the authors as spatial scattering modulation (SSM), which uses indices of scatterer clusters to deliver additional information bits. By viewing the indices of the scatterer clusters as the indices of radiation beams projected towards the scatterers, the SSM appears similar to the BIM concept presented in this paper. However, the SSM proposed in [6] only works for a single user uplink scenario, because the SSM receiver needs to be equipped with multiple RF chains in order to perform maximum likelihood (ML) detection. This will ultimately limit its applications in future device-to-device communication systems, e.g., the Internet of Things, [7].

To address this problem, for the BIM scheme presented in this paper, the information recovery strategy is significantly simplified, so that only a single RF chain is required at the receiver side, thus extending applications to low-power low-cost systems. The simplified receiver architecture and its associated decoding strategy also result in enhanced system performance, compared with the ML detection used in the SSM scheme.

This paper is organized as follows; In Section 2 the BIM architecture and its operational principle are elaborated. The BIM bit error rate (BER) simulations under various channel conditions are presented and compared with those in SSM and classical beamforming systems in Section 3, from which the guidelines for system designers are discussed. In Section 4, the beam-space channel measurement is conducted in a typical indoor environment at $10 \mathrm{GHz}$, and the results are used to calculate system BER performance, validating the design guidelines. Finally, Section 5 concludes the paper.

\section{Proposed beam index modulation systems}

The proposed BIM architecture is illustrated in Fig. 1. Both transmitter and receiver employ a beamforming 
network, so that a single RF chain, when routed through a reconfigurable switch array to one of its beam ports, can enable all of the available beamforming gain towards a corresponding spatial direction.

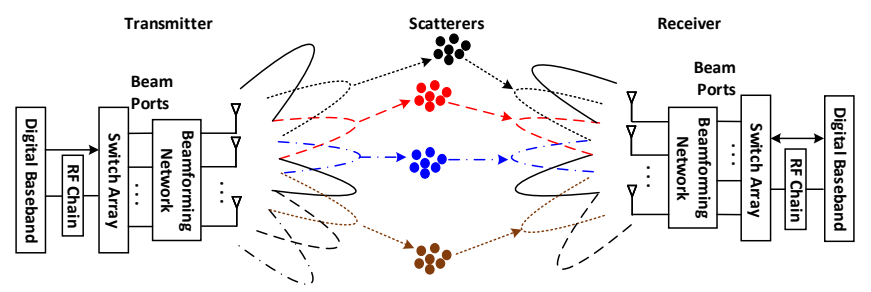

Fig. 1. Illustration of BIM system in sparse wireless propagation channels.

It is assumed that before the data transmission occurs, the system is trained in beam-space [8], [9]. Due to the training required, the proposed technique only suits temporally static channel scenarios or with relatively long channel coherence time. During this beam training stage, the transmitter and receiver obtain knowledge of the indices of transmit and receive beams, which, through the relay of scatterers, enable propagation links with sufficient path gains. For the example shown in Fig. 1, the transmitter and the receiver need to know the indices of four transmitreceive beam pairs and their associated path gains. No beams are reused in different pairs. The guidelines for selecting beam pairs to construct BIM systems for enhanced performance are provided in the next section. In this paper, flat-fading channels are considered, so that the path gains can be represented by complex numbers.

After the beam training is performed, the BIM transmitter projects a modulated RF carrier through one of the selected transmit beams that have been obtained at the training stage. The index of the chosen beam is updated at the symbol rate and is determined by the baseband data of $\log _{2}(M)$ bits, where $M$ is the total number of selected beam pairs. Instead of receiving signals from each of selected receive beams to perform ML detection as used in SSM systems, which requires $M$ receive RF chains, the proposed BIM receiver simply detects the power at each of the $M$ beam ports of the receive beamforming network corresponding to the selected receive beams, to estimate the index of the activated transmit beam and connects the single receive RF chain to the beam port with the strongest power. Commonly available power detectors, e.g., [10], could be used for this purpose. In this way, information bits conveyed through transmit beam indices and RF carriers can be recovered at the receiver side. In this paper it is assumed that the power detectors suffer the same amount of noise as the receive chain discussed, meaning that the signal to noise ratios (SNRs) for beam indices detection and modulated data recovery are identical. In the next section, besides comparing the performance of the proposed BIM system with that of its SSM counterpart, single-RF-chain beamforming which transmits information always through the strongest propagation link is also used as a benchmark.

\section{Performance simulation and design guidelines}

In this section, extensive BIM system BER simulations are presented, and compared with those obtained through SSM and classical beamforming systems.
Following these studies, recommendation guidelines on how to choose beam pairs in order to construct BIM systems can be obtained.

First, some system assumptions are made, and associated parameters are defined;

- In the beam training stage, the $M$ beam pairs with highest path gains are identified, i.e., a channel matrix $\boldsymbol{H}$ of the size of $M$-by- $M$ in beam domain is known by both transmitter and receiver. Its entry $h_{i j}$ represents the transmission coefficient from the $i^{\text {th }}(i=1, \ldots, M)$ selected beam port of the beamforming network at the transmitter side to the $j^{\text {th }}(j=1, \ldots, M)$ selected beam port of the beamforming network at the receiver side. To facilitate discussion and formulation, it is assumed that $\left|h_{m m}\right| \geq\left|h_{n n}\right|$ when $m<n$. Operator '|.' returns the modulus of the enclosed complex number. After assigning indices of the selected transmit and receive beams using the above rule, the diagonal entry $h_{m m}$ in $\boldsymbol{H}$ is the transmission gain of the $m^{\text {th }}$ propagation path, linking the transmitter and the receiver in the beam domain;

- The path gain of the strongest link is normalised to 0 $\mathrm{dB}$, namely $\left|h_{11}\right|=1$;

- The gain difference between the $m^{\text {th }}$ link and the strongest link, in $\mathrm{dB}$, is defined as

$$
\Delta g_{m}=20 \times \log _{10}\left(\left|h_{m m}\right| /\left|h_{11}\right|\right)=20 \times \log _{10}\left|h_{m m}\right| .
$$

To simplify the discussion, in this section it is assumed that $\Delta g_{m}$ for each $m(m \neq 1)$ are identical, and thus denoted as $\Delta g$;

- When the $i^{\text {th }}$ transmit beam is activated, there is some signal power leaked into the $j^{\text {th }}(j \neq i)$ receive beam if $h_{i j} \neq 0$. This beam crosstalk, in $\mathrm{dB}$, is defined as

$$
\Delta \beta_{i j}=20 \times \log _{10}\left(\left|h_{i j}\right| /\left|h_{i i}\right|\right) .
$$

Similarly, in this section it is assumed that $\Delta \beta_{i j}$ for each $i, j(i \neq j)$ combination are identical, and the subscript ' $i j$ ' can, thus, be omitted. When $\Delta \beta=-\infty$, we say beam pairs are orthogonal to each other;

- It is assumed that the channel noise presented at each beam port of the beamforming network at the receiver side is independent additive white Gaussian noise (AWGN), and they all follow $C N\left(0, \sigma^{2}\right)$;

- It is assumed that in the BIM system, the signal power injected into the selected transmit beam port is $P_{T x}$. A parameter, characterising the transmit power and receive noise per beam port, is defined, $S N R_{T x}=$ $10 \times \log _{10}\left(P_{T x} / \sigma^{2}\right)$

- When comparing the BER performance of the proposed BIM and the classical beamforming systems the transmission rates are kept identical. For example, the BIM data rate $\log _{2}(M)+\log _{2}(U)$ equals the beamforming data rate $\log _{2}(V)$, where $U$ and $V$ are, respectively, the modulation orders of the signals carried by RF carriers in the BIM and the beamforming systems. In the classical beamforming system, the transmitter and receiver always choose the strongest link in beam domain, i.e., $h_{11}$, for data transmission. For fair comparison, the mean consumed power at transmitter sides is made 
identical. It should be pointed out that it does not mean the signal power at the first transmit beam port in beamforming systems is $P_{T x}$ because different crest factors of modulated RF carriers in different systems are associated with different power efficiencies of transmit power amplifiers;

- For each BER simulation, a random data stream of at least $10^{+7}$ symbols is generated, which allows BER down to $10^{-5}$ to be calculated. Details on the procedures used for these BER calculations can be found in [11].

In Fig. 2 the simulated BERs in three different systems, namely BIM, SSM, and beamforming, under the simplest scenario are plotted. Here in the beamforming system, 8PSK modulated signals are transmitted through the strongest propagation link with the normalised path gain of $\left|h_{11}\right|^{2}=1$. While in the BIM and SSM systems, since $M=2$ beam pairs are available, to achieve 3 bits per transmission, QPSK modulation scheme is adopted. Initially it is assumed that no crosstalk between beam pairs exists, i.e., $\Delta \beta=-\infty$, and two beam pairs have equal gains, i.e., $\Delta g=0$. Since both 8PSK and QPSK are constant envelope modulation types, the radiated power in the beamforming system is the same as that in the BIM system, i.e., $P_{T x}$.

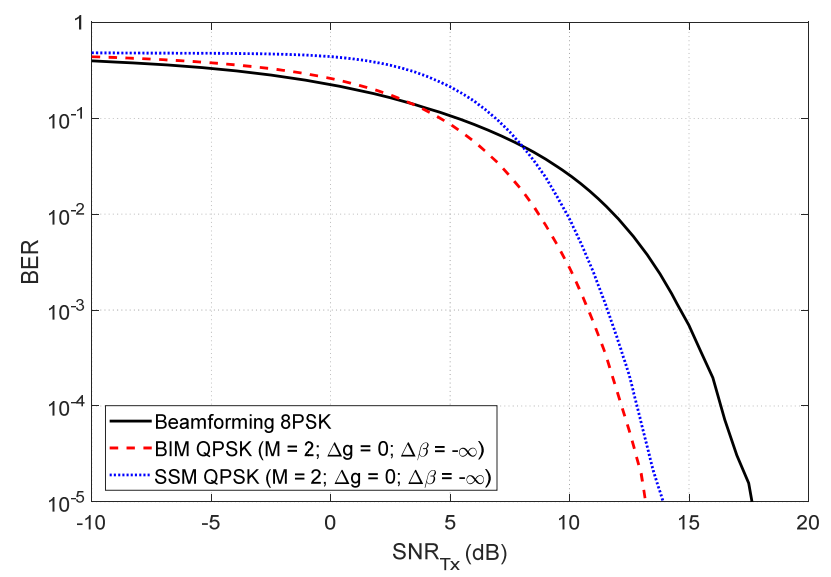

Fig. 2. Simulated BER performance in beamforming, BIM, and SSM systems. 8PSK modulation is used for beamforming, while in BIM and SSM, QPSK modulaiton is adopted, and it is assumed that $M=2, \Delta g=0$, and $\Delta \beta=$ $-\infty$.

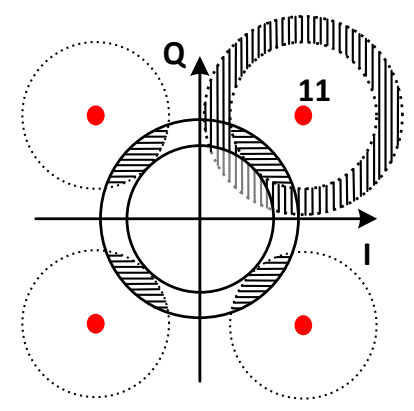

a

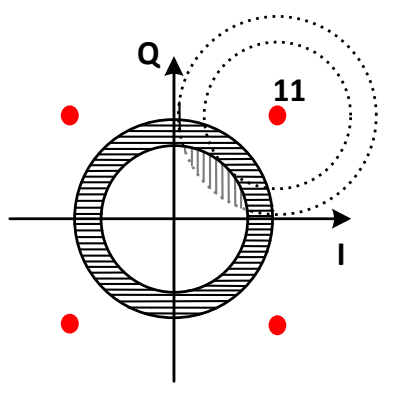

b
Fig. 3. Simplified graphical illustration for receivers of decoding signals incorrectly under the transmission scenario studied in Fig. 2.

(a) SSM receiver, (b) BIM receiver
Consistent with the findings in [6], it can be seen that the SSM performs better than the beamforming scheme when the orthogonal beam pairs are relatively balanced in terms of path gains. The proposed BIM system, surprisingly, despite of having only one RF chain at the receiver side, instead of two in the SSM receiver, outperforms SSM. This indicates the ML detection may not be the optimum strategy in decoding information bits that involve beam indices. This can be explained, with the help of graphical illustrations in Fig. 3, as follows;

- Since $M=2$, the SSM (or BIM) receiver only detects signals (or signal power) at the two selected receive beam ports;

- Since $\Delta g=0$, the expected QPSK constellation patterns in IQ planes presented at the two receive beam ports, after phase calibration is achieved in the beam training stage, are identical;

- For example, when the transmitter projects a QPSK symbol ' 11 ', in the first quadrant, through the second beam pair, the detected signal at the second receive beam port, after corruption by AWGN, follows a complex Gaussian distribution centred at the symbol ' 11 ' in the first quadrant. Since $\Delta \beta=-\infty$, the signal presented at the first receive beam port follows the same distribution, but centred at the origin in its IQ plane;

- In order to facilitate understanding, we draw two rings of the same size in the IQ planes associated with the two receive beam ports. These two rings are centred at their respect noiseless locations, i.e., the ring with the solid (or dotted) outline is centred at the origin (or the QPSK symbol '11') in the IQ plane at the first (or the second) beam port. For visual assistance the two IQ planes are overlapped;

- In the SSM system, the receiver performs the ML detection, i.e., the receiver translates the received signals into one of the reference symbols based on the minimum Euclidean distance in IQ planes. For the example discussed here there are eight reference symbols consisting of four QPSK symbols in IQ plane at the first beam port and the other four QPSK symbols in IQ plane at the second beam port. When only the received signals that fall into the two rings are considered, the shaded areas in Fig. 3(a), consisting of the horizontal and the vertical shaded areas for signals associated with the first and the second beam ports, respectively, correspond to cases where the ML receiver decodes the transmitted SSM symbol incorrectly. In Fig. 3(a), the three dotted circles of the same radius as the inner radius of the two rings are plotted in the other three quadrants in order to illustrate some decoding boundaries;

- In the proposed BIM system, the receiver first detects the signal power at the two beam ports, and only decodes the stronger signal, using the single RF chain, following the standard QPSK demodulation rule, i.e., demodulating received signals based on which quadrant in IQ plane they locate into. Thus, when the received signals that fall into the same two rings are considered, the areas that correspond to cases of an incorrect decode given by the BIM receiver are shaded in Fig. 3(b); 
- Since the two rings have the same radius, and the noise presented at the two receive beam ports are independent AWGN of the same power, the larger total shaded area in Fig. 3(a), compared with that in Fig. 3(b), indicates that higher BER occurs in the SSM system than in the proposed BIM system;

- The radii of the two rings can be altered to study other scenarios. The details can be different, but the conclusions are the same, i.e., the proposed BIM system outperforms its SSM counterpart.

In practical communication scenarios, the selected beam pairs are unbalanced $(\Delta g<0)$ and nonorthogonal $(\Delta \beta$ $\neq-\infty)$ to each other. The effects that these parameters have on the BER performance of the BIM system are first studied separately, see the simulation results in Fig. 4 and Fig. 5. As can be expected, the unbalanced path gains of the selected beam pairs and the crosstalk among them reduce the achievable BIM performance. In Fig. 6 the combined effects of these two parameters are presented. The shaded area refers to the selected two-beam-pair conditions, under which the two-beam-pair QPSK BIM system performs better than the single-beam 8PSK beamforming system. It should be noted that the requirement for relatively gain-balanced beam pairs normally cannot be satisfied in propagation channels where Line of Sight (LoS) links are available. This suggests that the proposed BIM system is the favourable choice in nLoS scenarios. On the other hand, the crosstalk between beam pairs is determined by both beamforming networks and the scatterer distributions in the propagation environment. It is expected that by employing Fourier beamforming networks, such as Butler matrix [12] and Fourier Rotman lens [13], which are able to generate orthogonal radiation beams along some discrete spatial directions, the chances of getting low beam pair crosstalk can be increased.

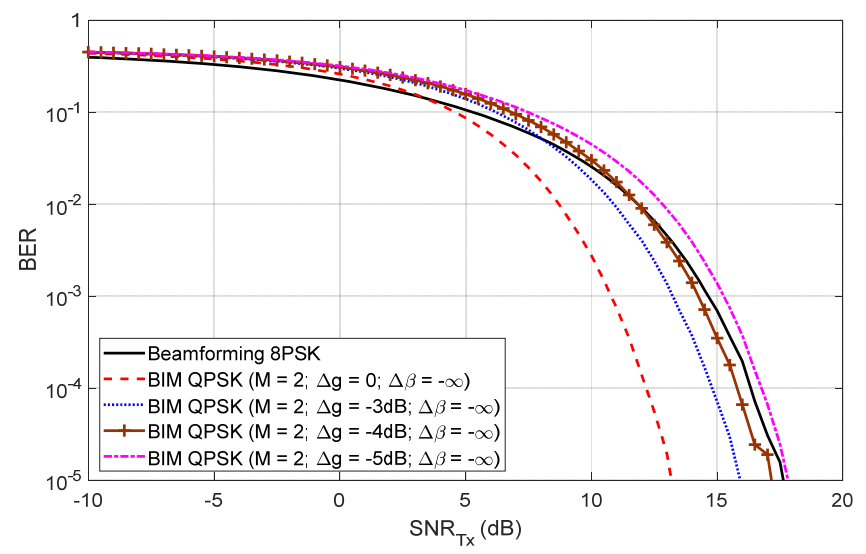

Fig. 4. Simulated BER performance in beamforming and BIM systems. 8PSK modulation is used for beamforming, while QPSK is used for BIM, and two $(M=2)$ path gain balanced $(\Delta g=0)$, or unbalanced $(\Delta g<0)$, orthogonal $(\Delta \beta$ $=-\infty)$ beam pairs are available.

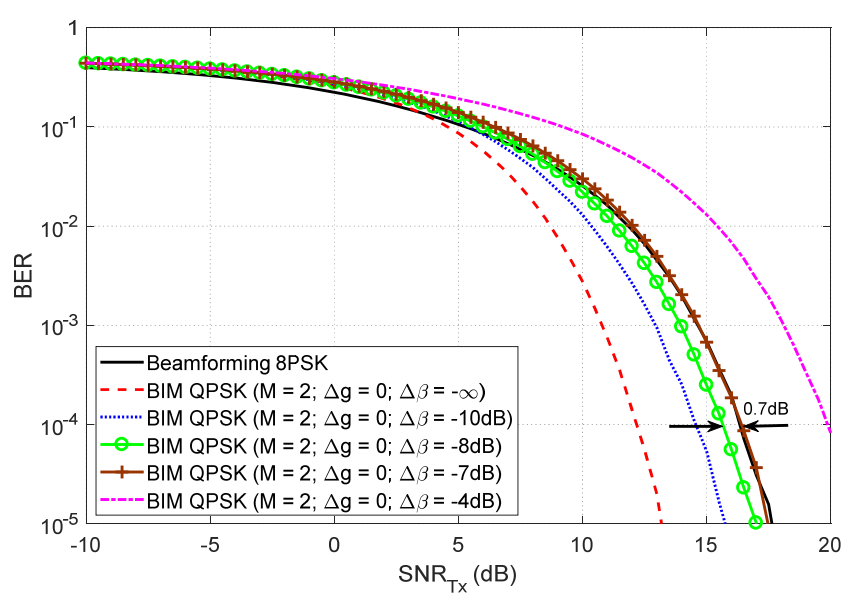

Fig. 5. Simulated BER performance in beamforming and BIM systems. 8PSK modulation is used for beamforming, while QPSK is used for BIM, and two $(M=2)$ path gain balanced $(\Delta g=0)$, orthogonal $(\Delta \beta=-\infty)$, or nonorthogonal $(\Delta \beta \neq-\infty)$ beam pairs are available.

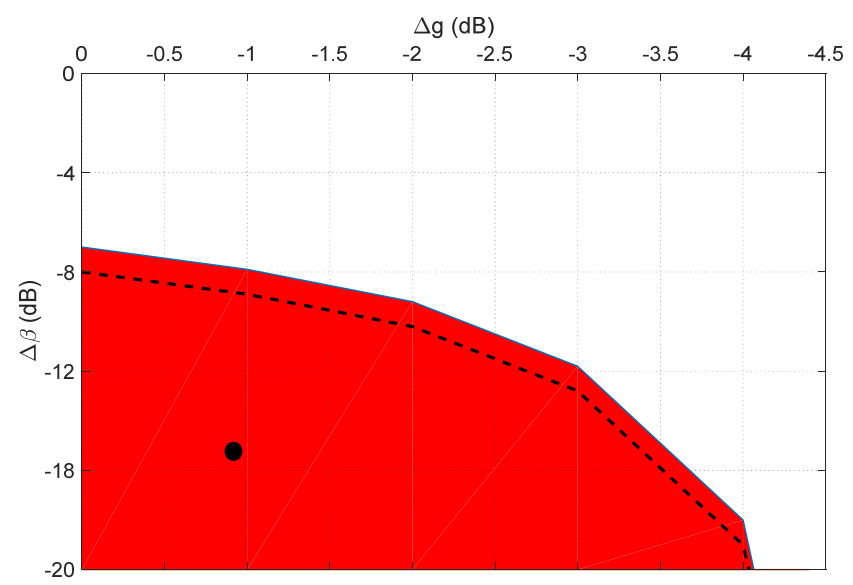

Fig. 6. The combined effects of $\Delta g$ and $\Delta \beta$ on the performance of the two-beam-pair QPSK BIM system. The shaded area refers to the channel conditions, under which the two-beam-pair QPSK BIM system performs better than the single-beam $8 P S K$ beamforming system.

When higher transmission rates are required, higher order modulation schemes should be adopted. For example, 16QAM beamforming systems enable four bits per transmission. In order to achieve the same transmission rate in this case, the radiated single RF carrier in the BIM and SSM systems with $M=4$ (or $M=2$ ) available beam pairs needs to be QPSK (or 8PSK) modulated. In this paper, only unfiltered signal waveforms are considered. Since 16QAM is not a constant envelope modulation scheme, and its peakto-average power ratio (PAPR) is $2.55 \mathrm{~dB}$, to avoid compression distortion, the operation point of the associated transmit power amplifier should be $2.55 \mathrm{~dB}$ back-off from its compression point, resulting in lower power efficiency. Take a typical two-way Doherty power amplifier in [14] as an example, its efficiencies at $\mathrm{P} 1 \mathrm{~dB}$ and $2.55 \mathrm{~dB}$ lower than $\mathrm{P} 1 \mathrm{~dB}$ are around $58 \%$ and $52 \%$, respectively. Thus, in order to maintain the same level of the mean power consumption at transmitter sides, the radiated power in the 16QAM beamforming systems is lower than that in the constantenvelope modulated BIM (or SSM) systems, i.e., $P_{T x_{-} 16 Q A M}$ $=(52 \% / 58 \%) \times P_{T x}$ in this example. When using these typical 
power amplifier efficiency values, the BER performance of the beamforming, SSM, and BIM systems for four bits per transmission is simulated under ideal beam pair conditions $(\Delta g=0$ and $\Delta \beta=-\infty)$, and they are shown in Fig. 7. Similar to the results in Fig. 2, we can see that the proposed BIM systems have better BER performance than their beamforming and SSM counterparts. In addition, when more ideal beam pairs are available, the lower order of the modulated RF carrier helps further performance enhancement, since further separated signal constellation points are more resilient to noise.

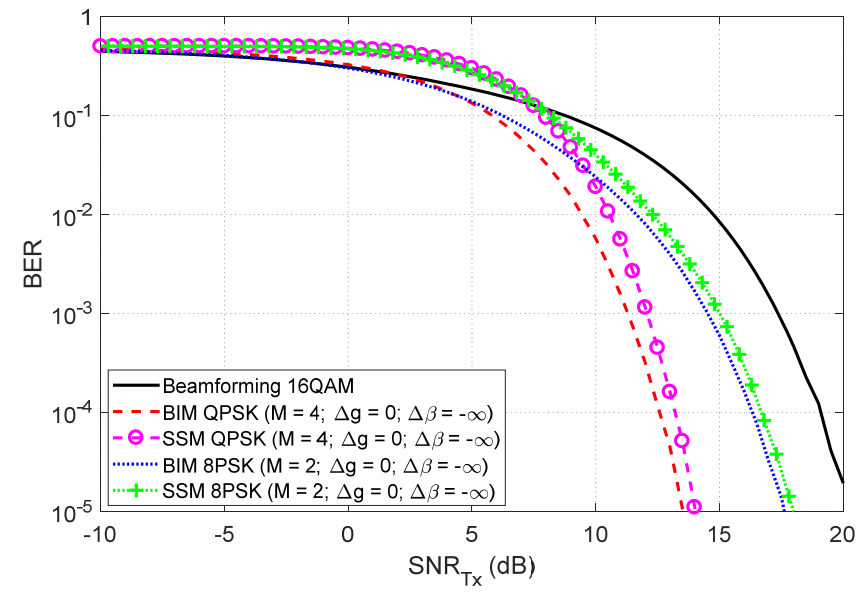

Fig. 7. Simulated BER performance in beamforming, BIM, and SSM systems. 16QAM modulation is used for beamforming, while in BIM and SSM, QPSK (or 8PSK) modulaiton is adopted when $M=4$ (or $M=2$ ). It is assumed that $\Delta g=0$, and $\Delta \beta=-\infty$.

In order to help system designers determine whether a transmitter should be configured for the proposed BIM or the classical beamforming, parametric studies need to be conducted, including the path gains of all available beam pairs, as well as crosstalk among them, and also the modulation schemes chosen for the radiated single RF carrier. In fact, when the modulation type for the RF carrier is selected, an enclosed $\left(M^{2}-1\right)$-dimension region, associated with each entry of the channel matrix $\boldsymbol{H}$ except the $h_{11}$ that acts as a reference, can be pre-generated and stored. When the channel parameters, obtained in the beam training stage, fall into this enclosed region, the transmitter should be configured for BIM transmission in order to achieve performance enhancement relative to classical beamforming which has a similar hardware complexity. It is pointed out that $h_{11}$ can only act as a reference when the beamforming system selects this link for transmission, otherwise the enclosed region needs to be shifted. This aspect is discussed with experimental data in Section 4. Here, to facilitate discussions and improve readability, following our assumptions that $\Delta g_{m}$ (or $\Delta \beta_{i j}$ ) for each $m, m \neq$ 1 , (or each $i, j$ combination, $i \neq j$ ) are identical, a 2-D region, similar to the one shown in Fig. 6, is obtained when the RF carrier in the 4-beam-pair BIM systems is QPSK modulated, see Fig. 8. The shaded area poses constraints on $\left(M^{2}-1=\right.$ 15) channel parameters in order for the BIM system to outperform the beamforming. In practice, it can be difficult to find a channel environment that satisfies all of these constraints. In this sense, the two-beam-pair 8PSK BIM can

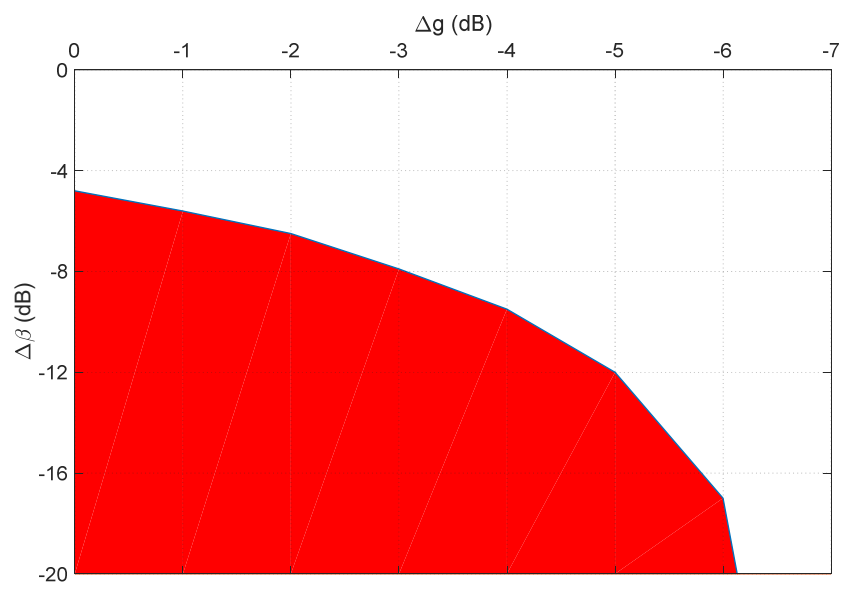

Fig. 8. The combined effects of $\Delta g$ and $\Delta \beta$ on the performance of the 4-beam-pair QPSK BIM system. The shaded area refers to the channel conditions, under which the 4-beam-pair QPSK BIM system performs better than the single-beam 16QAM beamforming system.

be a reasonable choice, since to perform better only $\left(M^{2}-1\right.$ = 3) channel parameters need to fall into a pre-stored enclosed region. This enclosed region for the two-beam-pair 8PSK BIM system is similar to the one shown in Fig. 6, and, thus, is omitted here.

\section{Experimental validation}

In this section, the experimental results for a beamspace channel in a typical indoor environment are provided. These are then used to calculate achievable BER performance if the beamforming, the SSM, and the proposed BIM systems are to be implemented. The results obtained validate the performance superiority of the BIM system when the beam pairs are properly selected following the design criteria articulated in this paper.
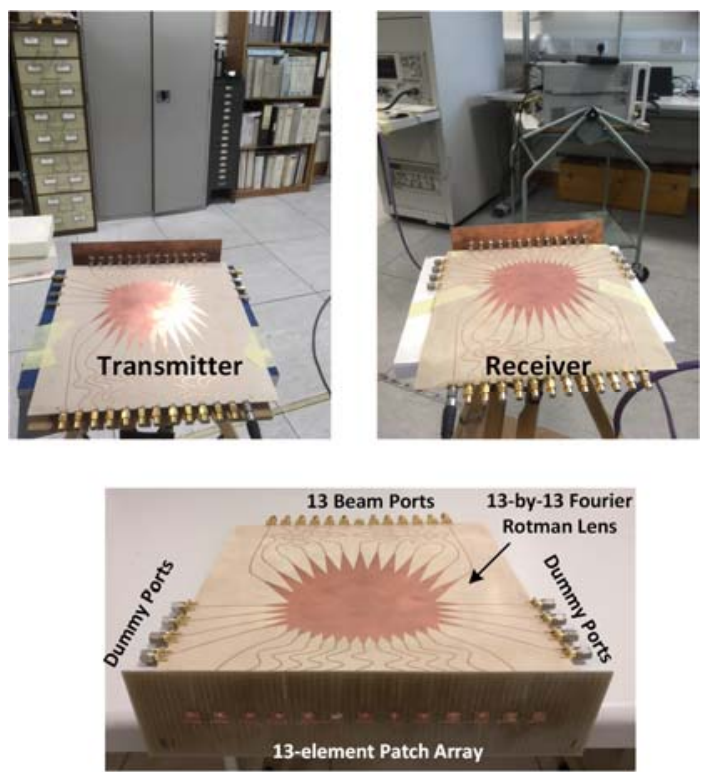

Fig. 9. A photograph of the fabricated Fourier Rotman lens and the linear array for $10 \mathrm{GHz}$ operation, and a photograph of the experimental setup in a typical indoor environment. 


\subsection{Experimental Setup and Measured Results}

Employing a 13-by-13 Fourier Rotman lens and a linear half-wavelength spaced microwave patch antenna array at both transmitter and receiver ends, the channel matrix in beam-space was measured at $10 \mathrm{GHz}$ in a typical indoor multipath environment. Boresight of the transmit and the receive antenna arrays were not aligned, but were pointed to some metallic scatterers in order to create more exploitable nLoS links. In addition, the LoS link was deliberately blocked. The design details of the Fourier Rotman lens and the antenna array, as well as their measured performance, can be found in [15]. A photograph of the experimental setup is shown in Fig. 9.

The 13-by-13 beam-space channel matrix was measured, and the power of its entries $h_{i j}$ are plotted in Fig. 10 .

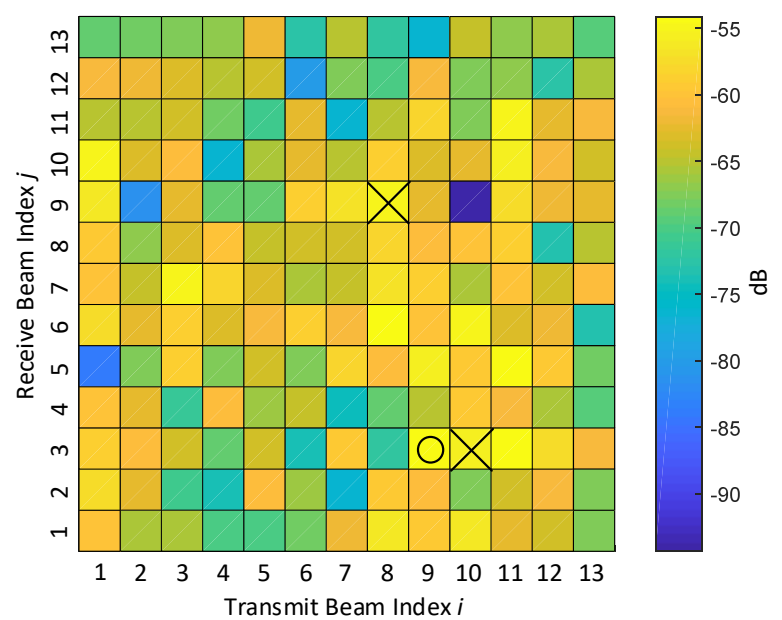

Fig. 10. The power of measured 13-by-13 channel matrix in beam domain at $10 \mathrm{GHz}$ in the typical indoor environment shown in Fig. 9.

\subsection{System Performance Prediction and Calculation}

The strongest link between the transmitter and the receiver is $(i=9, j=3)$, circled in Fig. 10, with the measured gain of $-54.1 \mathrm{~dB}$. If the beamforming strategy is to be adopted this link should always be used. It is normalised to $0 \mathrm{~dB}$ and is written as $h_{b f}$. On the other hand, to select beam pairs to construct the proposed BIM, the gains of all selected beam pairs need to be high and the

Table 1 Measured gains of the propagation links in beamspace between the transmitter and the receiver, which are selected for beamforming and two-beam-pair BIM systems

\begin{tabular}{|c|c|c|c|c|c|c|}
\hline \multicolumn{3}{|c|}{$\begin{array}{l}\text { Gains of two selected transmit- } \\
\text { receive beam pairs }(i, j) \text { and } \\
\text { their crosstalk for BIM (dB) }\end{array}$} & \multicolumn{4}{|c|}{$\begin{array}{l}\text { The normalised beam-space channel } \\
\text { matrix } \boldsymbol{H}_{B I M}\end{array}$} \\
\hline & \multicolumn{2}{|c|}{$i$} & \multirow{2}{*}{\multicolumn{2}{|c|}{$20 \log _{10}\left(\left|h_{B I M m n}\right|\right)$}} & \multicolumn{2}{|c|}{$m$} \\
\hline & 8 & 10 & & & 1 & 2 \\
\hline \multirow{2}{*}{$j$} & -54.8 & -94.3 & \multirow{2}{*}{$n$} & 1 & -0.7 & -40.2 \\
\hline & -71.9 & -55.7 & & 2 & -17.8 & -1.6 \\
\hline \multicolumn{3}{|c|}{$\begin{array}{c}\text { The strongest link }(i=9, j=3) \\
\text { has a gain of }-54.1 \mathrm{~dB}\end{array}$} & \multicolumn{4}{|c|}{$20 \log _{10}\left(\left|h_{b f}\right|\right)=0$} \\
\hline
\end{tabular}

crosstalk among them need to be low. There can be various beam pair solutions, one of which is labelled with crosses in Fig. 10. Here two beam pairs are selected and their measured values, including gains and crosstalk, are listed in Table 1. The normalised beam-space channel matrix for the BIM strategy is expressed as $\boldsymbol{H}_{B I M}$, with its entries $h_{B I M\{1,2\}\{1,2\}}$ also shown in Table 1 .

Since the strongest link $h_{b f}$ is not selected for the BIM system due to high crosstalk, i.e., $20 \log _{10}\left(\left|h_{99}\right| /\left|h_{93}\right|\right)=$ $-8.2 \mathrm{~dB}$, the graph in Fig. 6 that is generated based on the case of $\left|h_{b f}\right|=\left|h_{B I M 11}\right|$, as we discussed in Section 3, cannot be used directly. Since $20 \log _{10}\left(\left|h_{b f}\right| /\left|h_{B I M 11}\right|\right)=-0.7 \mathrm{~dB}$, and we know that when $\Delta g=0, \Delta \beta$ is required to be reduced from $-7 \mathrm{~dB}$ to $-8 \mathrm{~dB}$ for the two-beam-pair QPSK BIM system having the same BER performance as that of the 8PSK beamforming system to compensate this $0.7 \mathrm{~dB}$ SNR loss, see Fig. 5, before Fig. 6 can be applied for the experiment example with values in Table 1 , the boundary in Fig. 6 needs to be shifted downwards by $1 \mathrm{~dB}$, see the dashed line there. After the boundary correction, from the values in Table 1, we can get that

$$
\Delta g=20 \log _{10}\left(\left|h_{B I M 22}\right| /\left|h_{B I M 11}\right|\right)=-0.9 \mathrm{~dB}
$$

and

$$
\Delta \beta \leq 20 \log _{10}\left(\left|h_{B I M 12}\right| /\left|h_{B I M 11}\right|\right)=-17.1 \mathrm{~dB} .
$$

Therefore, the BER performance of the QPSK BIM system, if constructed using the two selected beam pairs in Table 1, is expected to be better than that of the QPSK BIM system associated with the dot in Fig. 6. Since it falls well in the shaded area, it outperforms the beamforming system, see the BER simulations in Fig. 11.

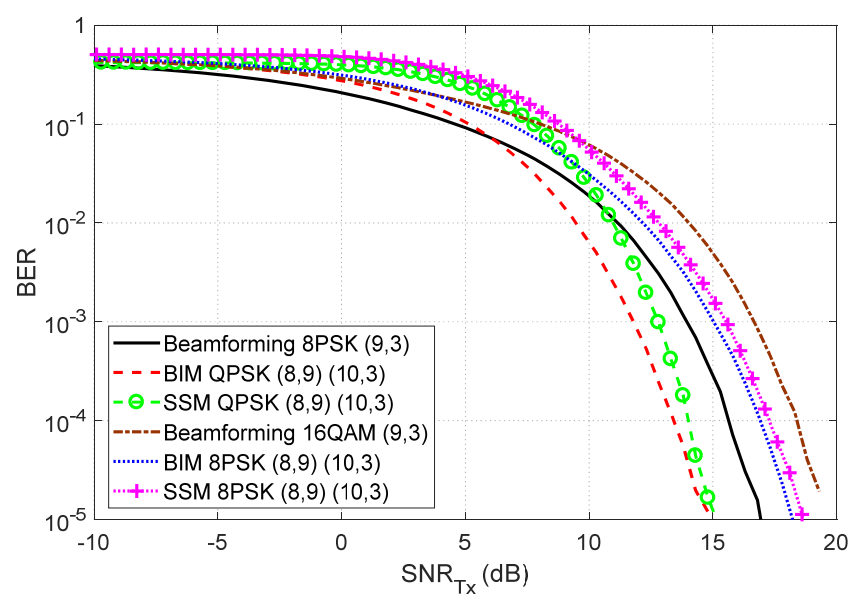

Fig. 11. The calculated BER performance in beamforming, SSM, and BIM systems based on the selected beam pairs ( $i$, $j)$, labelled in the legend, with the measured data in Table 1.

As we pointed out in Section 3 that, in practice, it can be difficult to have more than 2 beam pairs whose parameters fall into a per-stored enclose region to endow enhanced BIM performance over the beamforming system, and this is true for the measured channel matrix in Fig. 10. As a result, we shall use two beam pairs and 8PSK modulated RF carrier for BIM systems if 4 bits per transmission are required. As expected, the relatively 
balanced beam pairs and low crosstalk make the two-beampair 8PSK BIM system a better choice over the 16QAM beamforming system, see Fig. 11. Furthermore, the proposed BIM also outperforms SSM, despite only a single $\mathrm{RF}$ chain being used at the receiver side.

\section{Conclusion}

This paper proposed a new wireless transmission scheme, BIM, which uses activated beam indices to convey extra information bits, in addition to the information modulated onto the RF carriers. In this way, to transmit the same amount of information bits in each channel use as the beamforming system that has a similar hardware complexity, the BIM transmitter is able to choose lower modulation orders. This paper investigated the channel conditions, in beam-space, under which the BIM systems can achieve enhanced BER performance against their beamforming counterparts. It also shown that the BIM systems outperform previous SSM systems, with a less complex receiver architecture. In general, BIM systems work better in nLoS sparse wireless channels such as exist in indoor environment when operation frequencies are high. The parametric studies, presented in this paper, provided the guidelines for system designers to configure the transmitter based on the channel knowledge obtained in the beam training stage. This aspect was validated by some calculations based on the measured channel matrix.

\section{Acknowledgments}

This work was supported by the EPSRC (UK) under Grants EP/N020391/1 and EP/P000673/1.

The authors thank Mr. Kieran Rainey for the help on the channel measurements.

\section{References}

[1] Bliss, D., Forsythe, K., Chan, A.: 'MIMO wireless communication', Lincoln Laboratory J., 2005, 15, (1), pp. 97-126

[2] Mesleh, R., Haas, H., Sinanovic, S., Ahn, C., Yun, S.: 'Spatial modulation', IEEE Trans. Veh. Technol., 2008, 57, (4), pp. 2228-2241

[3] Yang, P., Renzo, M., Xiao, Y., Li, S., Hanzo, L.: 'Design guidelines for spatial modulation', IEEE Commun. Surveys \& Tutorials, 2015, 17, (1), pp. 6-26

[4] Amadori P., Masouros, C.: 'Low RF-complexity millimeter-wave beamspace-MIMO systems by beam selection', IEEE Trans. Commun., 2015, 63, (6), pp. 2212-2223

[5] Renzo, M., Haas, H., Ghrayeb, A., Sugiura, S., Hanzo, L.: 'Spatial modulation for generalized MIMO: challenges, opportunities, and implementation', IEEE Proc., 2014, 102, (1), pp. 56-103

[6] Ding, Y., Kim, K., Koike-Akino, K., Pajovic, M., Wang, P., Orlik, P.: 'Spatial scattering modulation for uplink millimeter-wave systems', IEEE Commun. Lett., 2017, 21, (7), pp. 1493-1496

[7] Bello O., Zeadally, S.: 'Intelligent device-to-device communi-cation in the Internet of Things', IEEE Syst. J., 2016, 10, (3), pp. 1172-1182
[8] Dai F., Wu, J.: 'Efficient broadcasting in Ad Hoc wireless networks using directional antennas', IEEE Trans. Parallel Dist. Sys., 2006, 17, (4), pp. 335-347

[9] Wang, J., Lan, Z., Pyo, C., Baykas, T., Sum, C., Azizur Rahman, M., Funada, R., Kojima, F., Lakkis, I., Harada, H., Kato, S.: 'Beam codebook based beamforming protocol for multi-Gbps millimeter-wave WPAN systems', IEEE J. Sel. A. Commun., 2009, 27, (8), pp. 1390-1399

[10] 'HMC1094 data sheet', http://www.analog.com/media/ en/technical-documentation/data-sheets/HMC1094.pdf, accessed 30 August 2017

[11]Ding Y., Fusco, V.: 'Establishing metrics for assessing the performance of directional modulation systems', IEEE Trans. Antennas Propag., 2014, 62, (5), pp. 2745-2755

[12]Ding Y., Fusco, V.: 'Sidelobe manipulation using Butler matrix for $60 \mathrm{GHz}$ physical layer secure wireless communication', Proc. Antennas Propag. Conf., Loughborough, U.K., Nov. 11-12, 2013, pp. 61-65.

[13]Zhang, Y., Ding, Y., Fusco, V.: 'Sidelobe modulation scrambling transmitter using Fourier Rotman lens', IEEE Trans. Antennas Propag., 2013, 61, (7), pp. 3900-3904

[14] 'A2T21H140--24SR3 data sheet', http://www.nxp.com /docs/en/data-sheet/A2T21H140-24S.pdf, accessed 30 August 2017

[15]Ding, Y., Zhang, Y., Fusco, V.: 'Fourier Rotman lens enabled directional modulation transmitter', Int. J. Antennas Propag., 2015, 2015, Article ID 285986, 13 pages 